\title{
ASORS beim DKK 2016: Neues zur onkologischen Rehabilitation
}

Die ständig zunehmende Bedeutung der Rehabilitation im Betreuungskonzept onkologischer Patienten wurde beim Deutschen Krebskongress (DKK) 2016 sehr deutlich: Zahlreiche Sitzungen waren psychosozialen und medizinischen Themen gewidmet, die in der Rehabilitation von Tumorpatienten wichtig sind. Wir sprachen mit Professor Rick, Bad-Wildungen, im Zusammenhang mit dem DKK über den Stand der Rehabilitationsmedizin.

? Weshalb nimmt die Bedeutung der onkologischen Rehabilitation so stark zu?

Prof. Dr. Oliver Rick: Die onkologische Rehabilitation wird immer wichtiger, da die Inzidenz von Tumorerkrankungen zunimmt und in den nächsten Jahren weiter ansteigen wird. Darüber hinaus werden moderne onkologische Therapien die Lebenserwartung der Patienten weiter verbessern. Damit werden auch Folgestörungen von onkologischen Therapien eher

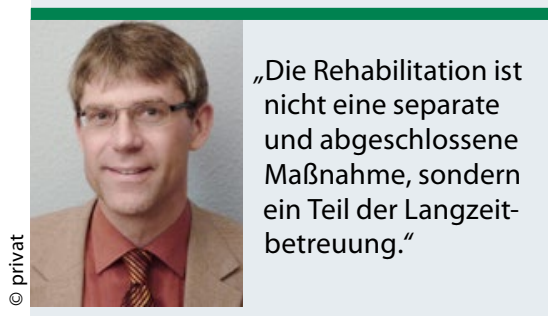

Prof. Dr. med. Oliver Rick, Bad Wildungen

Vorsitzender des Bereichs Rehabilitation der Arbeitsgemeinschaft Supportive Maßnahmen in der Onkologie, Rehabilitation und Sozialmedizin (ASORS) der Deutschen Krebsgesellschaft. präsent werden und entsprechende Behandlungskonzepte erfordern.

? Was sind die Ziele der Rehabilitation onkologischer Patienten?

Rick: Die Rehabilitation soll die Teilhabe der Patienten am Erwerbs- und Sozialleben gewährleisten. Dies gelingt uns, indem wir Patienten auf die Probleme nach onkologischen Therapien aufmerksam machen, ihnen Behandlungskonzepte anbieten und somit eine eigenständige Weiterbehandlung im persönlichen Umfeld ermöglichen.

? Sollte die Rehabilitation indikationsspezifisch oder fachübergreifend erfolgen? Rick: Sie sollte im Wesentlichen fachübergreifend erfolgen, da wir eine Vielzahl von Diagnosen haben. Folglich sind viele Fachdisziplinen im medizinischen, aber auch im paramedizinischen Bereich einzubinden, um einer großen Anzahl an Folgestörungen onkologischer Erkrankungen auf fachlich hohem Niveau Rechnung tragen zu können. Das bildet auch die S3Querschnittsleitlinie zu speziellen Rehabilitationsthemen $a b$, die zurzeit von der ASORS erarbeitet wird.
? Welche Patienten benötigen ganz besondere Maßnahmen?

Rick: Einerseits Patienten, bei denen eine Rückkehr ins Erwerbsleben im Raum steht. Hier muss ein besonderes Konzept im Rahmen der medizinisch-beruflich orientierten Rehabilitation durchgeführt werden. Andererseits Patienten mit schwerwiegenden Folgen der Antitumortherapie. Dazu gehören z.B. Patienten mit Lungenteilresektion oder mit Schluckstörungen nach Operationen von Kopf-HalsTumoren und Patienten mit Magenresektion, die das Essen wieder neu lernen müssen. Nach längerem Krankenhausaufenthalt sind ebenfalls besondere Maßnahmen notwendig. Ebenso für diejenigen Patienten, die unter psychischen Folgestörungen der Erkrankung und Therapie leiden.

? Was waren für Sie die Highlights beim Krebskongress aus Perspektive eines Rehabilitationsmediziners?

Rick: Ein Highlight war die Möglichkeit, auf die Bedeutung von onkologischer Rehabilitation als Startpunkt des Cancer Survivorships hinzuweisen und aufzuzeigen, dass rehabilitative Aspekte einen wertvollen Beitrag leisten können. Es wurde gezeigt, dass die Rehabilitation nicht als separate und abgeschlossene Maßnahme zu verstehen ist, sondern einen Teil der Langzeitbetreuung von Krebspatienten darstellt.

! Das Interview führte Petra Ortner zen oder Fatigue bestimmte Medikationsmanagementmodule durchliefen.

Das sorgte dafür, dass mögliche Probleme früh erkannt wurden. Unabhängig vom Alter der Patienten habe sich dadurch das Auftreten schwerer unerwünschter Wirkungen im Mittel um einen Therapiezyklus verzögern lassen, so Jaehde. Im nächsten Schritt soll das Bonner Medikationsmanagement jetzt um onkogeriatrische Assessments erweitert werden. Philipp Grätzel von Grätz

\section{Lungenkrebs: Auch im Alter stadienspezifisch behandeln}

Neun von zehn Lungenkrebspatienten sind 60 Jahre und älter, $43 \%$ über 75 . Wie so oft sind ältere Patienten in Therapiestudien auch in diesem Fall unterrepräsentiert. Für Nihilismus und Altersdiskriminierung ist aber auch beim Lungenkarzinom kein Platz. Das Alter an sich sei beim Lungenkrebs kein prognostisch relevanter Faktor, wohl aber die Komorbiditäten, betonte Sylvia Gütz,
Leipzig. Erfasst werden können sie z.B. mit dem Carlson Comorbidity Index (CCI), der den Einfluss der Begleiterkrankungen gewichtet nach dem Einfluss auf die Sterblichkeit bewertet. In einer Studie mit 1.255 Patienten mit nichtkleinzelligem Lungenkarzinom (NSCLC) erhöhte ein CCI-Wert von 1 und mehr gegenüber einem Wert von 0 das Mortalitätsrisiko signifikant (Hazard Ratio [HR] 1,28; $95 \%$-Konfidenzintervall [95\%-KI] 1,09-1,5; $\mathrm{p}=0,003)$, nicht 